\title{
Penetrating neck injuries
}

\author{
B. Vishwanatha - A. Sagayaraj · Shalini G. Huddar · Prashanth kumar · R. K. Datta
}

\begin{abstract}
The incidence of penetrating and lacerated neck injuries has been rising in recent decades largely because of urban violence. Injury to the neck frequently results in multiple regional injuries and in addition poses serious threat to vital structures in the neck.
\end{abstract}

From 1999 to 2005, forty-two cases of penetrating neck injuries which were treated in our hospital were included in this study. Thirty one (73.8\%) injuries were due to homicide, six cases (14.2\%) were due to suicide attempt and five $(11.9 \%)$ were accidental injuries. Surgical management included tracheostomy neck exploration and wound repair. All the patients were followed up for a minimum period of six months. Six patients $(14.2 \%)$ had unilateral vocal cord paralysis. Two patients (4.7\%) developed tracheal stenosis.

A proper evaluation, rapid air way intervention and proper surgical repair are essential for a successful outcome.

Keywords Penetrating neck injuries - Tracheostomy

B. Vishwanatha ${ }^{1}(\bowtie) \cdot$ A. Sagayaraj ${ }^{2}$ S. G. Huddar ${ }^{2} \cdot$ P. kumar $^{2}$ '

R. K. Datta ${ }^{2}$

${ }^{1}$ Asst. Professor, Department of E.N.T.

Victoria Hospital and Bangalore Medical College

Bangalore - 560002 ,

Karnataka,

India.

${ }^{2}$ Post Graduates, Department of E.N.T.

Bangalore Medical College

\# 716, 10th Cross, 5th Main,

M.C. Layout, Vijayangar,

Bangalore - 560 040,

Karnataka

India.

E mail: drbviswanatha@yahoo.co.in

Ph: +91 / $80 / 23381567$

Mobile: +91 / 0 / 9845942832

\section{Introduction}

All penetrating neck injuries are potentially dangerous and require emergency treatment because of the presence of vessels, nerves and organs in the neck [1]. For the head and neck surgeon the management of these injuries is crucial. The exact documentation of these injuries can be very useful to determine whether the injury was homicidal or self inflicted [2].

Difficulties in evaluation and management of penetrating neck injuries is due to the complicated anatomy of the area, in which dense concentration of vital vascular, aero digestive, and nervous system are located in a very small space.

Thorough knowledge of the anatomy of neck, physical assessment and diagnostic and therapeutic interventions are necessary for appropriate management. Expeditious decision making is often required to prevent catastrophic airway, vascular, or neurological sequelae.

\section{Objectives}

To do a clinical study of penetrating neck injuries.

\section{Patients and methods}

From 1999 to 2005, forty-two cases of penetrating neck injuries, which were treated in our hospital are included in this study. Surgical management included tracheostomy neck exploration and wound repair. All the patients were followed up for a minimum period of six months.

\section{Ohservations}

The patients presenting were in the range of 18 to 70 years and mean age of presentation was 32 years. Thirty four patients were males and eight were females. Six patients had injuries in zone I, thirty four patients had injuries in zone II and only two patients had injuries in zone III (Table 1). All patients presented with wound in the neck. 
Common symptoms were haemorrhage, stridor and subcutaneous emphysema. Thirtyone injuries were due to homicide (Fig. 1), six cases were due to suicide attempt (Fig. 2) and five were accidental injuries (Fig. 3).

Forty patients underwent tracheostomy, neck exploration and wound repair. All cases were operated under general anesthesia. Laryngeal mucosal lacerations were repaired.Glottic and supra glottic lacerations were approximated and repaired. Displaced laryngeal cartilage fractures were approximated surgically. Two patients had oesophgeal injury and it was repaired. Two patients had associated pneumothorax.Two patients had injuries in zone III with
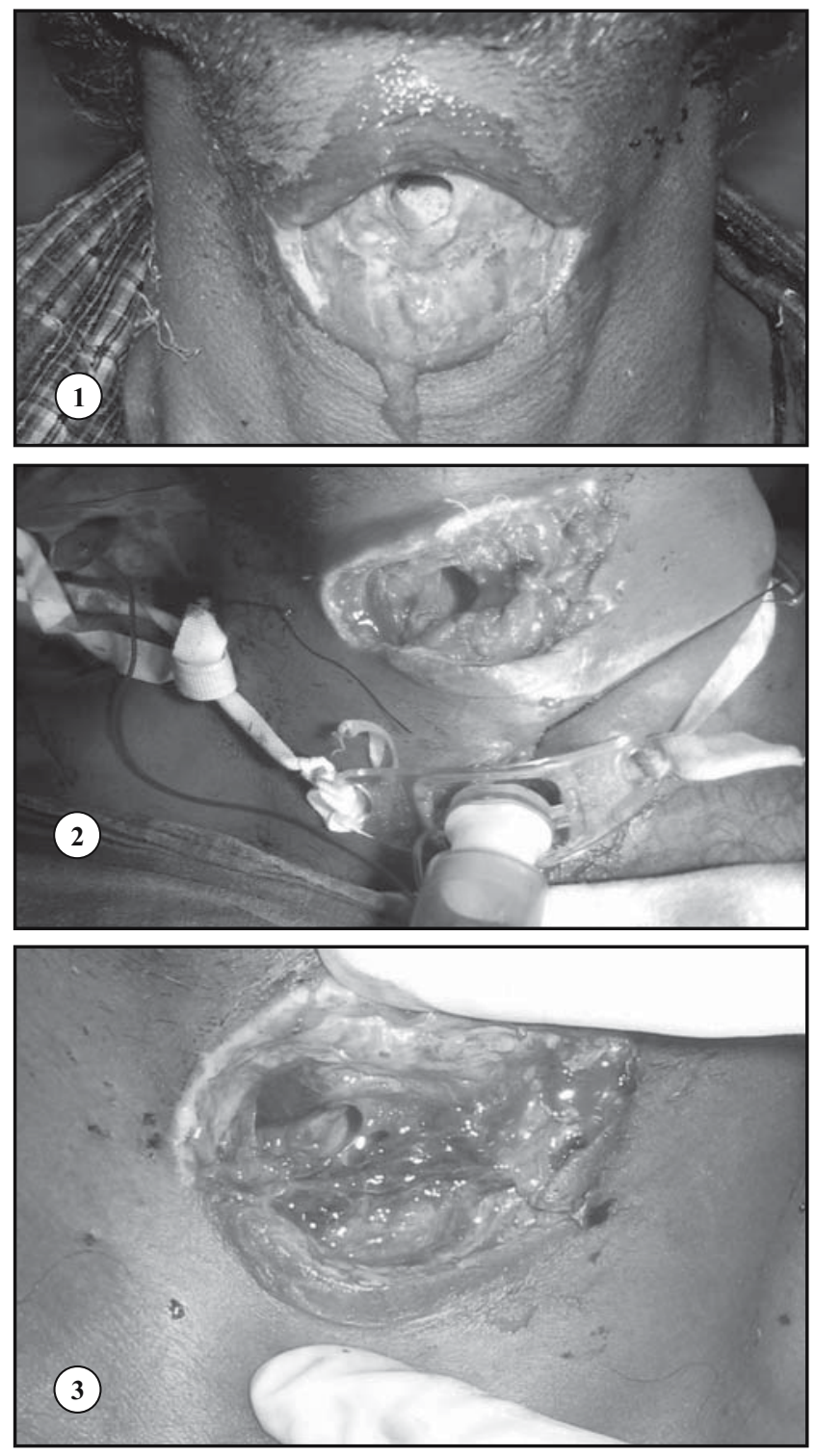

Fig. 1 Photograph showing homicidal neck wound

Fig. 2 Photograph showing self inflicted neck wound with tracheostomy tube

Fig. 3 Photograph showing accidental neck wound stable vital signs. These patients were kept under observation and medical line of management was done. Table 2 shows neck structures injured in forty two patients. There was no mortality.

All the patients were followed up for a minimum period of six months. Six patients had unilateral vocal cord paralysis. Two patients developed tracheal stenosis.

\section{Discussion}

Penetrating neck injuries are presented in $5-10 \%$ of all trauma cases. Many kinds of material can cause penetrating trauma to the neck, such as a knife, shotgun, pencil and fragments of glass and metal [1].

Before the second world war, few penetrating wounds under went operative treatment unless major bleeding or deep injuries were obvious; reported mortality rates were $11 \%$ in World War I. Fogelman and Stewart in1956 reported a mortality rate of $6 \%$ with prompt exploration versus $35 \%$ in cases with delayed or omitted operation. This led to the wide spread theory of treating the platysma like peritoneum: if violated explore. Later during World War II, management changed to mandatory exploration of all neck wounds [3].

Multiple vital structures of the neck which are vulnerable to injury can be divided into four groups: [1]

(i) the air passages: trachea, larynx and lung;

(ii) vascular structures: carotid, jugular, subclavian, innominate and aortic arch vessels;

(iii) gastrointestinal structures: pharynx and oesophagus; and

(iv) neurological structures: cranial nerves, peripheral nerves, brachial plexus and spinal cord.

Penetrating neck injuries are usually described in terms of their location in one of three anatomic zones as described by Monson [3, 4].

Zone I is the region that extends from the inferior border of the cricoid cartilage to the clavicles. Injuries in this region carry a high mortality risk because they involve the thoracic structures, major vessels, upper mediastinum, lungs, trachea, esophagus, and thoracic duct, and because surgical exposure is difficult.

Zone II is the area between the cricoid cartilage and the angle of the mandible. This is the most frequently injured region of the neck but the mortality rate from these injuries is relatively low because of better surgical exposure compared to other zones. Underlying structures are the major arteries and veins of the neck, the air and food passages, the cervical spine, and the thyroid gland.

Zone III comprises the area between the angle of the mandible and the base of the skull. Wounds in this region are most commonly associated with vascular and pharyngeal injuries. As in zone I, surgical exposure is difficult in this area. 
Table 1 Injury according to zones, sex and mode of injury

\begin{tabular}{lllllll}
\hline Neck zone & Male & Female & Homicide & Self-inflicted & Accident & Total \\
\hline Zone I & $4(9.5 \%)$ & $2(4.7 \%)$ & $6(14.2 \%)$ & 0 & 0 & $6(14.2 \%)$ \\
Zone II & $28(66.6)$ & $6(14.2 \%)$ & $24(57.1 \%)$ & $6(14.2 \%)$ & $4(9.5 \%)$ & $34(80.9 \%)$ \\
Zone III & $2(4.7 \%)$ & 0 & $1(2.3 \%)$ & 0 & $1(2.3 \%)$ & $2(4.7 \%)$ \\
Total & $34(80.9 \%)$ & $8(19 \%)$ & $31(73.8 \%)$ & $6(14.2 \%)$ & $5(11.9 \%)$ & $42(100 \%)$ \\
\hline
\end{tabular}

Table 2 showing structures injured in the neck

\begin{tabular}{|c|c|c|c|}
\hline S1 no & Organ injured & No. of cases $(n=42)$ & Percentage \\
\hline 1 & Tracheal injury & 6 & $14.2 \%$ \\
\hline 2 & Oesophagus injury & 2 & $4.7 \%$ \\
\hline 3 & Cricoid cartilage injury & 2 & $4.7 \%$ \\
\hline 4 & Thyroid cartilage injury & 12 & $28.5 \%$ \\
\hline 5 & Arytenoid cartilage injury & 6 & $14.2 \%$ \\
\hline 6 & Thyroid gland injury & 4 & $9.5 \%$ \\
\hline 7 & Thyrohyoid membrane injury & 18 & $42.8 \%$ \\
\hline 8 & Hyoid bone injury & 2 & $4.7 \%$ \\
\hline 9 & Pharynx injury & 9 & $21.4 \%$ \\
\hline 10 & Submandibular gland injury & 2 & $4.7 \%$ \\
\hline 11 & Vocal cord paralysis & 6 & $14.2 \%$ \\
\hline
\end{tabular}

Although zones I and III are protected by bones, penetrating trauma of these area is more dangerous than in zone II because of proximity of thorax and skull base. The vital structures in the zone II are not protected by bone, and the risk of injury is different to that in zone I and III [1].

Knowledge of the common consequences of each zone of injury gives some idea of the most likely injuries, the probability of urgent airway management problems, and any expected surgical intervention. [4]. However, any patient with obvious signs or symptoms of a major vascular or tracheoesophageal injury, regardless of anatomical zone, should be emergently explored [3].

The neck is divided into two major triangles by sternocleidomastoid muscle, the anterior and posterior. The anterior cervical triangle contains submandibular, submental, superior and inferior carotid triangles. The submandibular triangle is outlined by the two converging bellies of the digastric muscle and the inferior margin of the mandible. The submandibular triangle contains the submandibular gland, lymphatic structures, parts of the anterior facial vein and facial artery, and the marginal mandibular branch of the facial nerve. The carotid triangle is outlined the posterior belly of the digastric muscle, the omohyoid muscle and sternocleidomastoid muscle. The carotid triangle carotid artery, jugular vein, hypoglossal and vagus nerves [1].

In addition to the above classifications, clinicians should recognize that wounds in the anterior and lateral aspects of the neck compromise the airway more often than those in the posterior region (behind sternocleidomastoid) because of their proximity to the larynx, trachea, laryngeal nerves, and cervical vessels. Lack of symptoms and absence of neck swelling do not exclude the possibility of underlying serious injury. Patients should be evaluated carefully and a plan should be made based on the zone injured, and the patient's stability. Because the neck is such a complex area, the evaluation should include the respiratory, cardiovascular, nervous, digestive and endocrine systems. [4]

Types of injury and associated findings can be classified as follows $[1,5]$ :

a. Findings suggestive of air way injury: Common signs of laryngeal injury include stridor, subcutaneous emphysema, hemoptysis, hematoma, ecchymosis, laryngeal tenderness, vocal cord immobility, loss of anatomical landmarks, and crepitus. Other findings may include fracture of the hyoid bone, widening of the thyrohyoid membrane, displaced fractures of the thyroid cartilage, fracture of the cricoid cartilage, and separation of the cricoid cartilage from the larynx or trachea.

b. Findings suggestive of pharyngo-oesophageal injury: dysphagia, soft tissue crepetus, enlarged retropharyngeal space, trachycardia

c. Findings suggestive of vascular injury: expanding haematoma thrills and bruits, hypovolemic shock, persistent hemorrhage.

d. Neurologic injury: hoarseness, cranial nerves deficits,

Evaluation of laryngeal trauma begins with ensuring a patent airway. There is no uniform agreement as to the preferred method of airway management in patients with penetrating neck injuries. The preferred artificial airway in patients with laryngeal injuries is a tracheostomy, which should be performed with the patient awake under local 
anesthesia if possible. Associated cervical spine, esophageal, and vascular injuries can then be evaluated. Most authors agree that blind intubation methods (i.e. blind nasotracheal intubation) should not be used in these patients because further injury or complete airway obstruction may be induced. A blind attempt at intubating the trachea may carry the risk of introducing the tracheal tube into a false passage or cause a complete disruption of the endolaryngeal structures, or even facilitating laryngo-tracheal separation. The incidence of these phenomena is unknown but is most likely lethal and difficult to reverse even with an emergency surgical airway $[1,6]$.

The patient should be placed in Trendelenburg to decrease the risk of air embolism - a common cause of death in the presence of major venous disruptions [3].

Recommendations for diagnostic work-up and surgical management are quite variable in the medical literature. They range from mandatory operative intervention to selective management with evaluation of the vascular system (carotid and vertebral arteries, jugular veins, and subclavian vessels), the respiratory system (trachea and larynx), and the digestive system (esophagus and pharynx). Because of difficulty in surgical exposure, selective management is frequently chosen for injuries in zones I and III if the patient is stable and has no obvious injuries. Operative management includes a thorough exploration of the neck that involves visualization of the esophagus, pharynx, carotid artery, jugular veins, neural structures, airway (larynx and trachea), and injury tract [4].

The goal of treatment is to restore the 3 primary functions of the larynx- airway, voice, and swallowing, and to prevent complications such as airway embarrassment, dysphonia, tracheal/laryngeal stenosis, and dysphagia.

Laryngeal mucosal lacerations from penetrating injury should be repaired early (with in 24 hours).Significant glottic and supra glottic lacerations and displaced cartilage fractures need surgical approximation. In a study Leopold found that the time elapsed before repair has an effect on both airway stenosis and on voice [7].An associated oesophageal lesion requires immediate surgical repair given the risk of mediastinitis [8].

\section{Conclusion}

A proper evaluation, rapid air way intervention and proper surgical repair are essential for a successful outcome. Early management of laryngeal injury within first 24 hours seems to bear the best results for air way and voice. Aggressive surgery is indicated to prevent the complications such as hoarseness, laryngeal stenosis and dysphagia. Successful decanulation, airway patency, a functional voice and normal deglutinatination can be expected in majority of patients with laryngeal injuries who are managed with prompt air way management and proper surgical repair. Finally, head and neck surgeons treating neck injuries should be familiar with the signs, symptoms and proper airway management.

\section{References}

1. Ozturk K, Keles Bahar, Cenik Z and Yaman H (2006) Penetrating zone II neck injury by broken windshield; International Wound Journal 3(1):63-66

2. Godbole BG, Vira TM, Rao RV (1980) Stab injury of neck; J Postgrad Med 26:257-258

3. Bardley J Phillips (2002) The penetrating neck wound: A few points. The internet journal of surgery. 3 (2)

4. Desjardins G and Varon AJ (2001) Airway management for penetrating neck injuries:the Miami experience.Resuscitation 48:71-75

5. Mandavia DP, Qualls S and Ivan (2000) Emergency airway management in penetrating neck injury. Annals of emergency medicine 35:221-225

6. Verschueren DS, Bell RB, Bagheri SC, Dierks EJ and Potter BE (2006) Management of laryngo- tracheal injuries associated with craniomaxillofacial trauma. Journal of Oral and Maxillofacial Surgery 64:203-214

7. Leopold DA (1983) Laryngeal trauma :A historical comparison of treatment methods. Arch Otolaryngol 109: $106-110$

8. Andres AG, Herrero PA, Diez FJ et al (2005) Medical and surgical management of noniatrogenic traumatic tracheobronchial injuries. Arch Bronconeumol 41:249-254 